\title{
Prometastatic mechanisms of CAF-mediated EMT regulation in pancreatic cancer cells
}

\author{
TAO SHAN ${ }^{1 *}$, SHUO CHEN $^{1 *}$, XI CHEN $^{1}$, WAN RUN LIN ${ }^{2}$, WEI LI $^{3}$, JIANCANG MA $^{1}$, \\ TAO WU ${ }^{1}$, HONG JI ${ }^{1}$, YIMING LI ${ }^{1}$, XIJUAN CUI ${ }^{4}$ and YA'AN KANG $^{5}$
}

\begin{abstract}
${ }^{1}$ Department of General Surgery, The Second Affiliated Hospital of Medical College, Xi'an Jiaotong University, Xi'an, Shaanxi 710004; ${ }^{2}$ Department of Pathology, Fudan University Shanghai Cancer Center, Shanghai 200032; ${ }^{3}$ Graduate School, Fourth Military Medical University, Xi'an, Shaanxi 710033; ${ }^{4}$ Department of General Surgery, First Affiliated Hospital of Medical College, Xi'an Jiaotong University, Xi'an, Shaanxi 710061, P.R. China; ${ }^{5}$ Department of Surgical Oncology, The University of Texas MD Anderson Cancer Center, Houston, TX, USA
\end{abstract}

Received July 27, 2016; Accepted November 14, 2016

DOI: 10.3892/ijo.2016.3779

\begin{abstract}
Tumor metastasis are accompanied by the EMT (epithelial-mesenchymal transition)-MET (mesenchymalepithelial transition) two-step process. In this study, we investigated the importance of cancer associated fibroblasts (CAF) in the process. First, the primary cultures of isolated pancreatic CAF, fibroblasts of normal pancreatic tissues (NF), and normal hepatic stellate cells (HSF) were identified and verified via the expression of $\alpha$-SMA and vimentin. Using an indirect three-dimensional co-culture model, the morphological changes were observed by light microscopy and laser scanning confocal microscopy. The invasive and migration capacity of pancreatic cancer cells was determined by Transwell chamber migration assay or scratch assay. The mRNA and protein expression levels of E-cadherin, vimentin, and Gli1 were determined by RT-PCR and western blotting. Primary cultures of isolated CAF, NF, HSF showed satisfactory growth with active proliferation. Indirect co-culture with CAF, BxPc-3 and Panc-1 cells showed significant partial EMT, reduced E-cadherin expression, and enhanced vimentin expression as compared with the single culture and NF/HSF co-culture groups, with corresponding increases in migratory and invasive capacities. PCR and western blotting results showed that mRNA and protein expression levels of Gli1 in $\mathrm{CAF}$ and Snail in cancer cells were increased. This process could be reversed by inhibition of hedgehog $(\mathrm{HH})$ signaling
\end{abstract}

Correspondence to: Dr Xi Chen, Department of General Surgery, The Second Affiliated Hospital of Medical College, Xi'an Jiaotong University, Xi'an, Shaanxi 710004, P.R. China

E-mail: chenxijiaoda@163.com

*Contributed equally

Key words: cancer associated fibroblasts, epithelial-mesenchymal transition, pancreatic cancer, hedgehog signaling in CAF. In the tumor microenvironment, activation of CAF is the key event in mediating partial EMT, and its mechanism may be associated with paracrine action after activation of $\mathrm{HH}$ signaling in CAF.

\section{Introduction}

Pancreatic cancer is a fatal malignant tumor that is often diagnosed at an advanced stage, and accompanied by invasion and metastasis (1). The metastasis of pancreatic cancer involves complex molecular mechanisms and hence, an accurate understanding of the mechanisms of pancreatic cancer metastasis is the prerequisite to an effective treatment for this disease.

Tumor metastasis refers to a multistep process involving tumor cell migration from the primary site to a distant site. This process includes: local invasion, intravasation, transportation, extravasation, and colonization (2). A study has shown that the re-induction of epithelial-mesenchymal transition (EMT) plays an important role in tumor metastasis (3). Although EMT is a critical enabler of metastasis in tumors with an epithelial origin, triggering EMT does not indicate that cells have entered an irreversible transition process (4). Thiery proposed a theory of two-step tumor metastasis, which states that the invasion and systemic spread of primary epithelial tumors are achieved by activating EMT (3). Three cell phenotypes exists during the process of EMT, specifically epithelial phenotype (E), mesenchymal phenotype (M) and partial EMT state $(\mathrm{P})$ which is an intermediate phenotype having both epithelial and mesenchymal features $(5,6)$. The tumor cells eventually form metastatic foci through a reverse process, transit back to epithelial phenotype, when disseminated to a distant site.

Currently, the exact mechanisms underlying key events for the spatiotemporal regulation of EMT-MET have not been elucidated (7). In pancreatic carcinomas, CAFs can compose up to $80 \%$ of the tumor mass (8). Also, in this study CAFs were found to secrete factors that enhance epithelial tumor cell proliferation and mutagenesis as well as angiogenesis (8). Literature has confirmed that activated cancer associated 
fibroblasts (CAF) can affect the promotion of tumor progression by EMT through the hedgehog $(\mathrm{HH})$ pathway (9).

We speculated that the state of fibroblasts may play an important role in the EMT-CTC (circulating tumor cell)-MET homing microenvironment. Therefore, our study utilized a three-dimensional co-culture to simulate the malignant interstitial tumor microenvironment, and observed the morphological changes of tumor cells during EMT using confocal laser scanning microscopy, RT-PCR, and western blotting. This study aims to comprehend the mechanism of pancreatic cancer metastasis from the perspective of the tumor microenvironment, and provide a new basis for therapeutic research.

\section{Materials and methods}

Materials. RIPA cracking liquid kits were obtained from Beyotime Biotechnology (Shanghai China). The DMEM culture medium and fetal calf serum were purchased from the Hyclone (USA). Transwell chambers were purchased from Millipore (Shanghai, China). Matrigel and One-Step RT-PCR kit were obtained from BD Biosciences (NJ, USA). E-cadherin, vimentin, Gli1, Snail and $\beta$-actin antibodies were purchased from the Santa Cruz Biotechnology (Santa Cruz, CA, USA). PTCH specific blocker were purchased from the Sigma Co. Ltd., Shanghai, China.

Cell cultures and treatments. The human pancreatic cancer cell lines (BxPc-3, Panc-1; obtained from the American Tissue Type Collection, USA) were maintained in Dulbecco's modified Eagle's medium (Gibco, USA) supplemented with penicillin $(100 \mathrm{U} / \mathrm{ml})$, streptomycin $(100 \mu \mathrm{g} / \mathrm{ml}), 0.1 \mathrm{mM}$ non-essential amino acids, $0.2 \mathrm{mM}$ glutamine, $1 \mathrm{mM}$ pyruvate, and $10 \%$ heat-inactivated fetal bovine serum and incubated in $5 \% \mathrm{CO}_{2}$ humidified atmosphere at $37^{\circ} \mathrm{C}$. Cells were grown to $80 \%$ confluency. In the invasion and migration experiments, the cells were cultured in Dulbecco's modified Eagle's medium without fetal bovine serum.

CAF cell separation, culture, and purification. Normal fibroblast (NF), CAF and hepatic stellate (HSF) cells were derived from patients with pancreatic cancer and hepatic trauma from the Second Affiliated Hospital of Xi'an Jiaotong University. All patients were newly diagnosed and had not received any relevant treatment prior to surgery. Informed consent was obtained from all patients before taking specimens. Fibroblast isolation was conducted as described previously (10). First, the tissue was trimmed to $1 \times 1 \times 1 \mathrm{~mm}$ and washed gently with PBS three times ( 5 min each). Next, the tissues were washed once with the medium and placed in fresh cell cultural medium containing $15 \%$ fetal calf serum, 2 mM L-glutamine, and $10 \%$ double-antibody. The tissue was cut with a sterile scalpel blade and sections of cells were gently scraped with a blunt blade. The cells were cultured in an incubator for $3-5$ days at $37^{\circ} \mathrm{C}$ and $5 \% \mathrm{CO}_{2}$. The medium was replaced once and every 3 days afterwards; after 14 days, the cells fully covered the petri dish. When the cell density reached $80-90 \%$, the cells were digested with trypsin and regenerated at a rate of 1:3. The CAFs and NFs used in the experiment were the 3rd and 5th generations of cells cultured in vitro, respectively, and showed no obvious aging phenotype.
Medium preparation of pancreatic CAFs, NFs and HSFs. CAFs were added to 6-well plates at a density of $1.5 \times 10^{5} / \mathrm{ml}$ and rinsed with PBS after $24 \mathrm{~h}$. The medium was replaced with serum-free medium and cultured for $48 \mathrm{~h}$, after which the culture broth was collected, centrifuged to remove the cells and debris, and the supernatant obtained was the CAF conditioned medium. These samples were stored at $4^{\circ} \mathrm{C}$. The conditioned medium of NFs and HSFs was collected in the same manner.

Indirect co-culture model of CAFs and pancreatic cancer cells. The pancreatic cancer cells BxPc-3 and Panc-1 were added to Petri dishes at a density of $1.5 \times 10^{5} / \mathrm{ml}$; after $24 \mathrm{~h}$, CAF-CM was added, and the cells were cultured for $48 \mathrm{~h}$. Cells in PBS or serum-free medium were used as controls. An inverted phase contrast microscope was used to observe the morphology and growth of pancreatic cancer cells in each Petri dish. Proteins were extracted from the cells. The co-culture of HSFs, NFs and pancreatic cancer cells was performed in the same manner.

Cell migration experiment. Cell migration capability was evaluated in a scratch test. BxPc-3 $\left(10 \times 10^{5}\right)$ and Panc-1 cells were seeded in $1.5 \mathrm{ml}$ media in each well into a 24 -well plate. The cells were grown to a confluent layer ( $48 \mathrm{~h}$ ), and then a scratch was made in each well by using a pipette tip. Subsequently, the cells were washed gently with PBS, and then culture broth of CAF, NF and F were added to the wells. Starting picture was taken at time-point 0 . The cells were then incubated at $37^{\circ} \mathrm{C}$ in a $5 \% \mathrm{CO}_{2}$ atmosphere, and new pictures were taken after $24 \mathrm{~h}$. The $24 \mathrm{~h}$ time-point was chosen to decrease the potential impact of proliferation on the closing of the scratch. Image-Pro Plus 5.0 from NIH was used to standardize and present the results.

Cell invasion experiment. Cell invasion was examined using Transwell assays, Following incubation for $48 \mathrm{~h}, 3 \times 10^{4}$ cells were transferred to the top of the Matrigel-coated invasion chambers (BD Biosciences, San Jose, CA, USA) in serum-free DMEM. DMEM containing 10\% FBS was added to the lower chamber. After $24 \mathrm{~h}$, the non-invading cells were removed, and the invading cells were fixed using $95 \%$ ethanol, stained with $0.1 \%$ crystal violet and images were captured at x100 magnification under an inverted phase contrast microscope (Olympus CKX31/41; Olympus, Tokyo, Japan). The experiments were repeated three times independently.

$R T-P C R$. Total RNA was extracted from the cells using TRIzol reagent. A total of $2 \mu \mathrm{g}$ RNA was reversed transcribed into first-strand cDNA using the Revert Aid First Strand cDNA Synthesis kit. PCR primer sequences were as follows: E-cadherin forward, 5'-CAATGGTGTCCATGTGAACA-3'; reverse, 5'-CCTCCTACCCTCCTGTTCG-3'. Vimentin forward, 5'-CGCTTCGCCAACTACAT-3'; reverse, 5'-AGGGCATC C ACTTCACAG-3'. $\beta$-actin forward, 5'-ATCGTGCGTGACAT TAAGGAGAAG-3'; reverse, 5'-AGGAAGGAAGGCTGGAA GAGTG-3'. Gli1 forward, 5'-ATAGTGAGCCATGCTGTC TCC-3'; reverse, 5'-TCTCTCTGGCTGCTCCATAACC-3'. Snaill forward, 5'-AAGGATCTCCAGGCTCGAAAG-3'; reverse, 5'-GCTTCGGATGTGCATCTTGA-3'. The PCR conditions were as follows: an initial reaction at $42^{\circ} \mathrm{C}$ for $1 \mathrm{~h}$ 


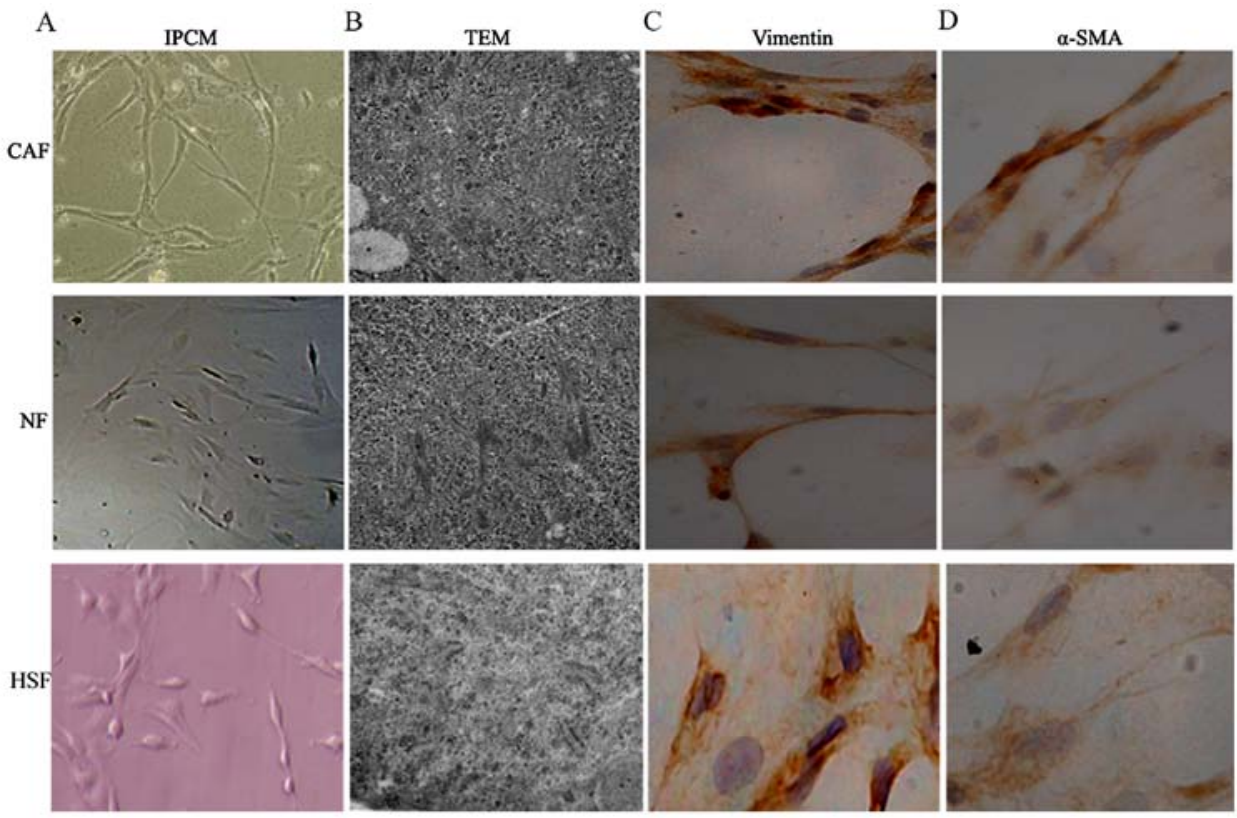

Figure 1. Preliminary identification of pancreas CAFs, NFs and HSFs. HSFs, NFs and CAFs showed a spindle shape. (A) The CAFs were spindle or fusiform shaped, had inconsistent sizes, showed dense growth, and were disorderly arranged. For NFs and HSFs, the cells showed multiple flat stellate shapes with similar cell sizes, were arranged in the same direction, and had a radially outward appearance. (B) TEM observations showed that CAFs had large cell nuclei, were evenly colored, and contained one or two nucleoli. In the cytoplasm, a large number of rough endoplasmic reticulum, mitochondria, and bundles of parallel subcapsular filaments were observed. For NFs and HSFs, the cells had an irregular cell nucleus, were rich in rough endoplasmic reticulum in the cytoplasm, and had no filaments. (C and D) Immunohistochemical results of CAFs showed that vimentin and $\alpha$-smooth muscle actin expression was positive In NFs and HSFs, vimentin showed positive expression, while $\alpha$-smooth muscle actin expression was negative.

was used for cDNA synthesis, followed by denaturation at $94^{\circ} \mathrm{C}$ for $5 \mathrm{~min}$ and 22 cycles of the following reactions: $94^{\circ} \mathrm{C}$ for $30 \mathrm{sec}, 55^{\circ} \mathrm{C}$ for $30 \mathrm{sec}$ and $72^{\circ} \mathrm{C}$ for $30 \mathrm{sec}$. After the last cycle, the reaction was amplified at $72^{\circ} \mathrm{C}$ for $10 \mathrm{~min}$. The housekeeping gene $\beta$-actin was used as an internal reference.

Western blotting. A total of $5 \times 10^{5}$ cells in the logarithmic growth phase was added to $0.5 \mathrm{ml}$ of pre-chilled cell lysis buffer and incubated on ice for $30 \mathrm{~min}$. After centrifugation, the supernatant was collected and the protein contents were measured. The proteins were separated by $10 \%$ SDS-PAGE and blotted onto a nitrocellulose membrane by semi-dry transfer. Next, the membrane was immersed in TBST containing 5\% skim milk for blocking followed by overnight incubation with the primary antibody at $4^{\circ} \mathrm{C}$. The following day, the membrane was incubated with the secondary antibody conjugated to horseradish peroxidase at 1:2,000 dilution (Santa Cruz) at room temperature for $2 \mathrm{~h}$, and then an enhanced chemiluminescence kit (Amersham Pharmacia Biotech, Amersham, UK) was used for staining. The membrane was photographed and the results were analyzed.

Statistical analysis. Each experiment was repeated at least three times. The data were expressed as mean \pm SD and analyzed using the Student's t-test and one-way ANOVA. $\mathrm{P}<0.05$ indicates a statistically significant difference.

\section{Results}

Preliminary identification of pancreas CAFs, NFs and $H S F$. After 10 days, cell morphology was observed using an inverted phase contrast microscope, HSFs, NFs and CAFs showed a spindle shape (Fig. 1). The CAFs were spindle- or fusiform-shaped, had inconsistent sizes, showed dense growth, and were disorderly arranged. For NFs and HSFs, the cells showed multiple flat stellate shapes with similar cell sizes, were arranged in the same direction, and had a radially outward appearance (Fig. 1). TEM observations showed that CAFs had large cell nuclei, were evenly colored, and contained one or two nucleoli. In the cytoplasm, a large number of rough endoplasmic reticulum, mitochondria, and bundles of parallel subcapsular filaments were observed. For NFs and HSFs, the cells had an irregular cell nucleus, were rich in rough endoplasmic reticulum in the cytoplasm, and had no filaments (Fig. 1). Immunohistochemical results of CAFs showed that vimentin and $\alpha$-smooth muscle actin expression was positive. In NFs and HSFs, vimentin showed positive expression, while $\alpha$-smooth muscle actin expression was negative (Fig. 1C and D).

Changes of partial EMT in co-cultured pancreatic cancer cells. To determine whether co-culture of pancreatic cancer cells with CAF promotes EMT, we first observed their morphology under conventional light microscopy. We found that after indirect co-culture with CAF, pancreatic cancer cells changed significantly from rounded to spindle-shaped or fusiform cells with a chaotic, non-directional arrangement, and radial appearance (Fig. 2A). Confocal laser scanning microscopy was carried out to assess the fluorescent intensity of Panc-1 cells. The results showed that pancreatic cancer cells co-cultured with CAF had a higher vimentin fluorescent intensity than the NF/HSF co-culture group and single culture group, whereas the E-cadherin fluorescent intensity was lower than the NF/HSF co-culture group and single culture group 

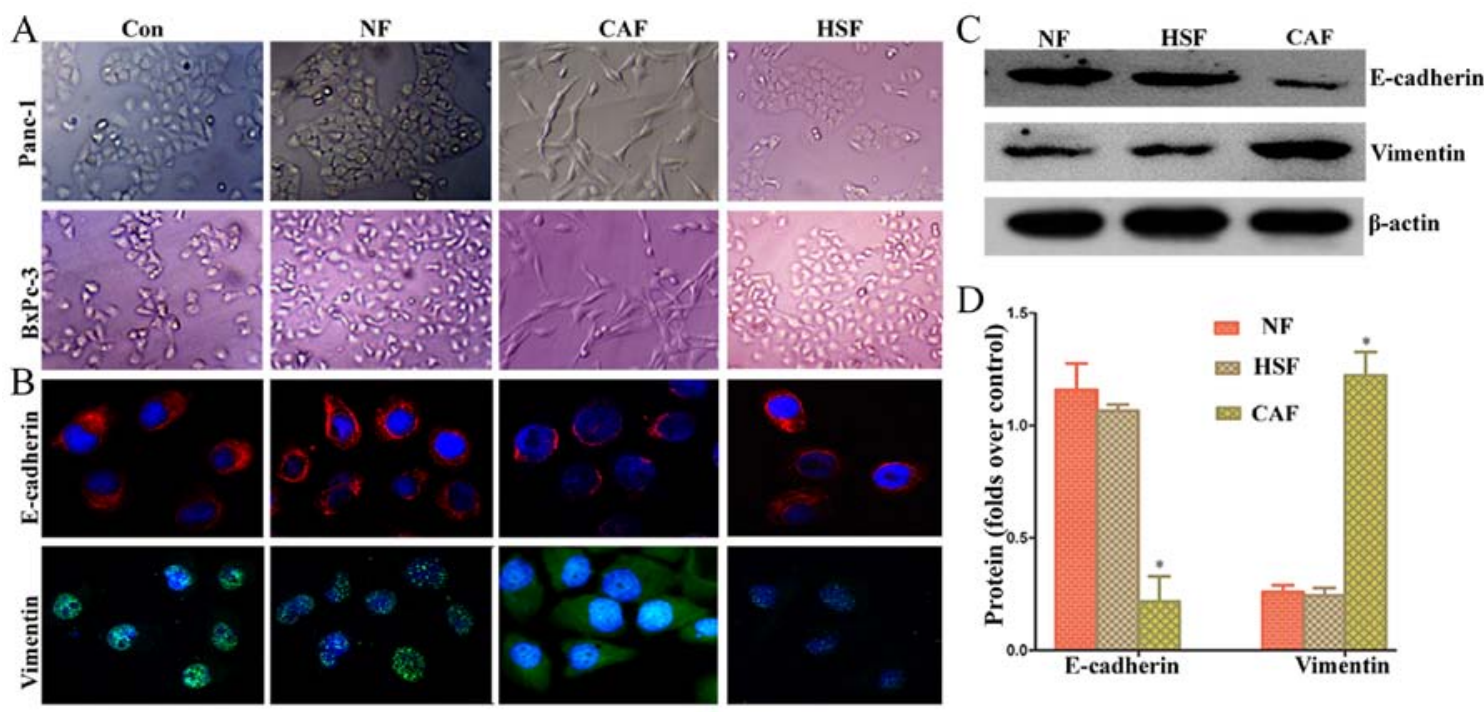

Figure 2. Changes of EMT in co-cultured pancreatic cancer cells. (A) After indirect co-culture with CAF, pancreatic cancer cells changed significantly from rounded to spindle-shaped or fusiform cells with a chaotic, non-directional arrangement, and radial appearance. was carried out to assess the fluorescent intensity of Panc-1 cells. (B) Confocal laser scanning microscopy showed that pancreatic cancer cells co-cultured with CAF had a higher vimentin fluorescent intensity than the NF/HSF co-culture group and single culture group, whereas the E-cadherin fluorescent intensity was lower than the NF/HSF co-culture group and single culture group. (C and D) Western blotting results showed that pancreatic cancer cells in the CAF co-culture group had a higher protein expression of vimentin than the NF/HSF co-culture group, whereas the protein expression of E-cadherin was lower than that in the NF/HSF co-culture group.
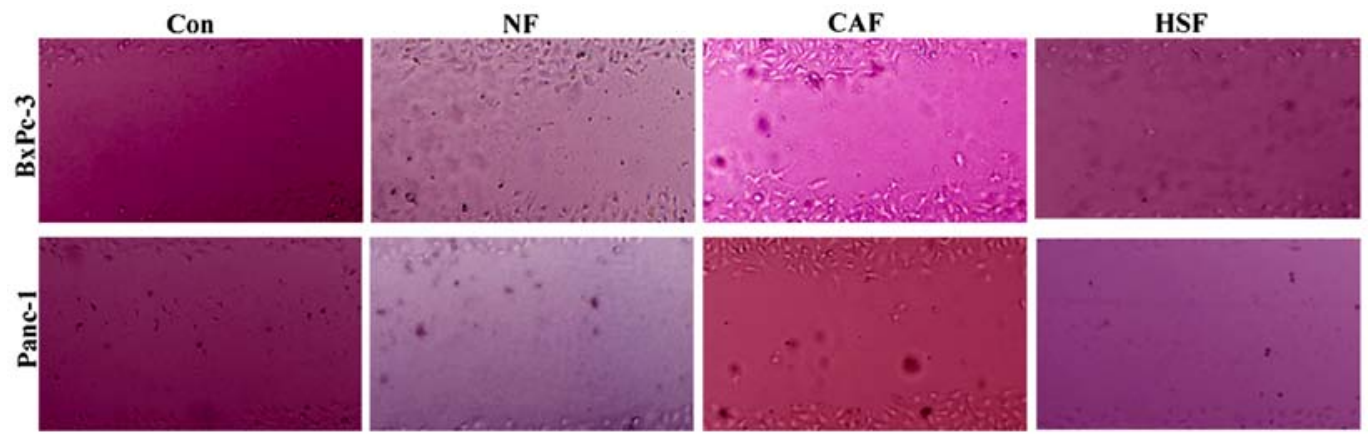

Figure 3. Changes in the migration capacity of co-cultured pancreatic cancer cells. BxPc-3 and Panc-1 cells were treated with CAF-conditioned medium (CM) for $48 \mathrm{~h}$, followed by a scratch assay to examine the effects of CAF on the migration of pancreatic cancer cells. After $48 \mathrm{~h}$ of treatment, single culture or NF/ HSF co-cultured BxPc-3 and Panc-1 cells exhibited weaker migratory capacity than the CAF co-culture group.

(Fig. 2B). Western blotting results showed that pancreatic cancer cells in the CAF co-culture group had a higher protein expression of vimentin than the NF/HSF co-culture group, whereas the protein expression of E-cadherin was lower than that in the NF/HSF co-culture group (Fig. 2C and D). The detection results of BxPc-3 cells were consistent with Panc-1 cells, suggesting that activated CAF can enhance EMT changes in pancreatic cancer cells, while EMT could not occur after co-culturing with quiescent HSF.

Changes in the migration capacity of co-cultured pancreatic cancer cells. To clarify whether CAF-induced partial EMT in pancreatic cancer cells can promote the pancreatic tumor migration, an indirect co-culture model was employed, where BxPc-3 and Panc-1 cells were treated with CAF-conditioned medium (CM) for $48 \mathrm{~h}$, followed by a scratch assay to examine the effects of $\mathrm{CAF}$ on the migration of pancreatic cancer cells. After $48 \mathrm{~h}$ of treatment, single culture or NF/HSF co-cultured BxPc-3 and Panc-1 cells exhibited weaker migratory capacity than the CAF co-culture group (Fig. 3). Results showed that CAF-induced partial EMT in pancreatic cancer cells enhancing pancreatic cancer cell migration; however, co-culture with quiescent HSF was unable to promote the migration of cancer cells.

Changes in the invasive capacity of co-cultured pancreatic cancer cells. To determine whether CAF-induced partial EMT in pancreatic cancer cells was able to promote the invasive capacity of pancreatic cancer cells, an indirect co-culture model was employed, followed by a Transwell migration assay using an NF-CM induction group and PBS alone as a control. Results showed that the CAF-CM treatment group had a significantly higher number of migrated cells than the PBS or NF/HSF-CM induction group (Fig. 4). This suggests that CAF-induced partial EMT in pancreatic cancer cells enhancing the invasive capacity of pancreatic cancer cells, whereas co-culture with quiescent HSF was unable to promote the invasive capacity. 

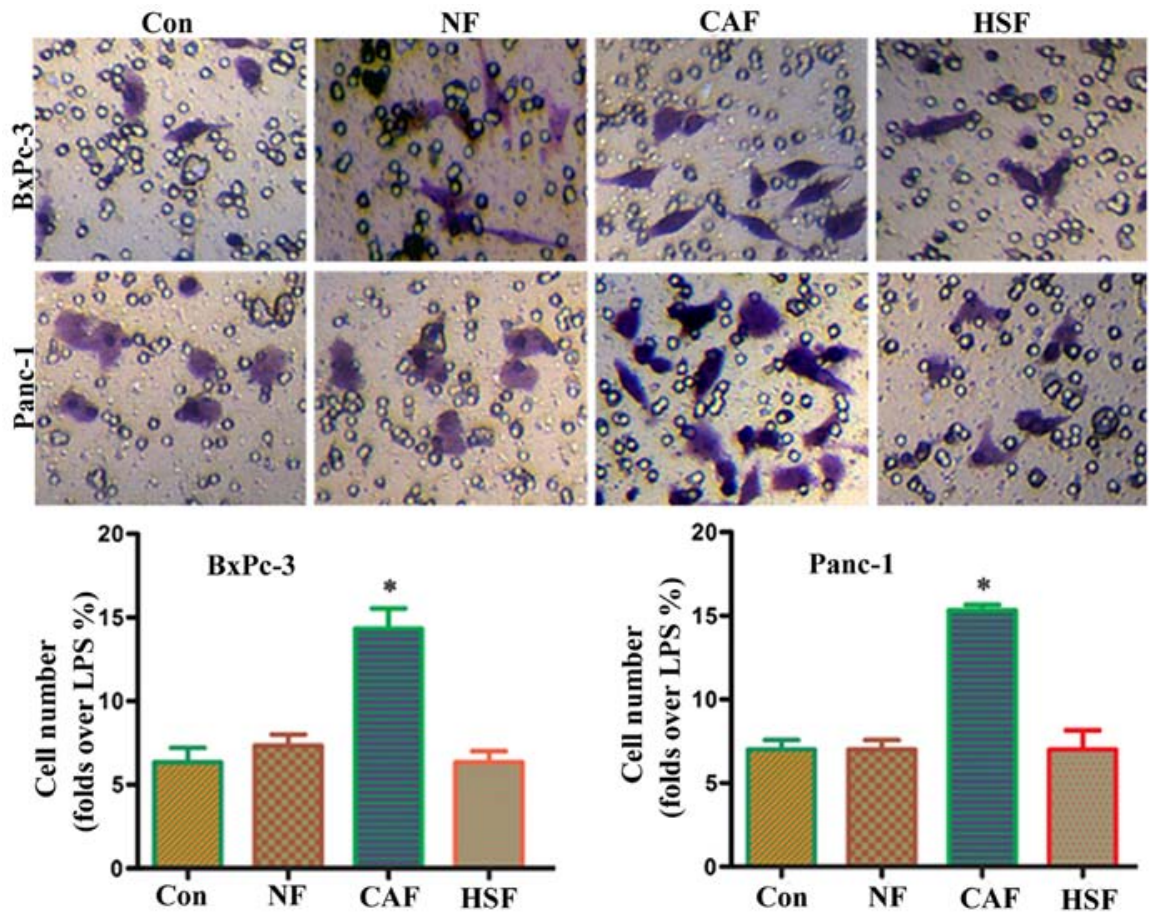

Figure 4. Changes in the invasive capacity of co-cultured pancreatic cancer cells. Results of Transwell migration assay showed that the CAF-CM treatment group had a significantly higher number of migrated cells than the PBS or NF/HSF-CM induction group.
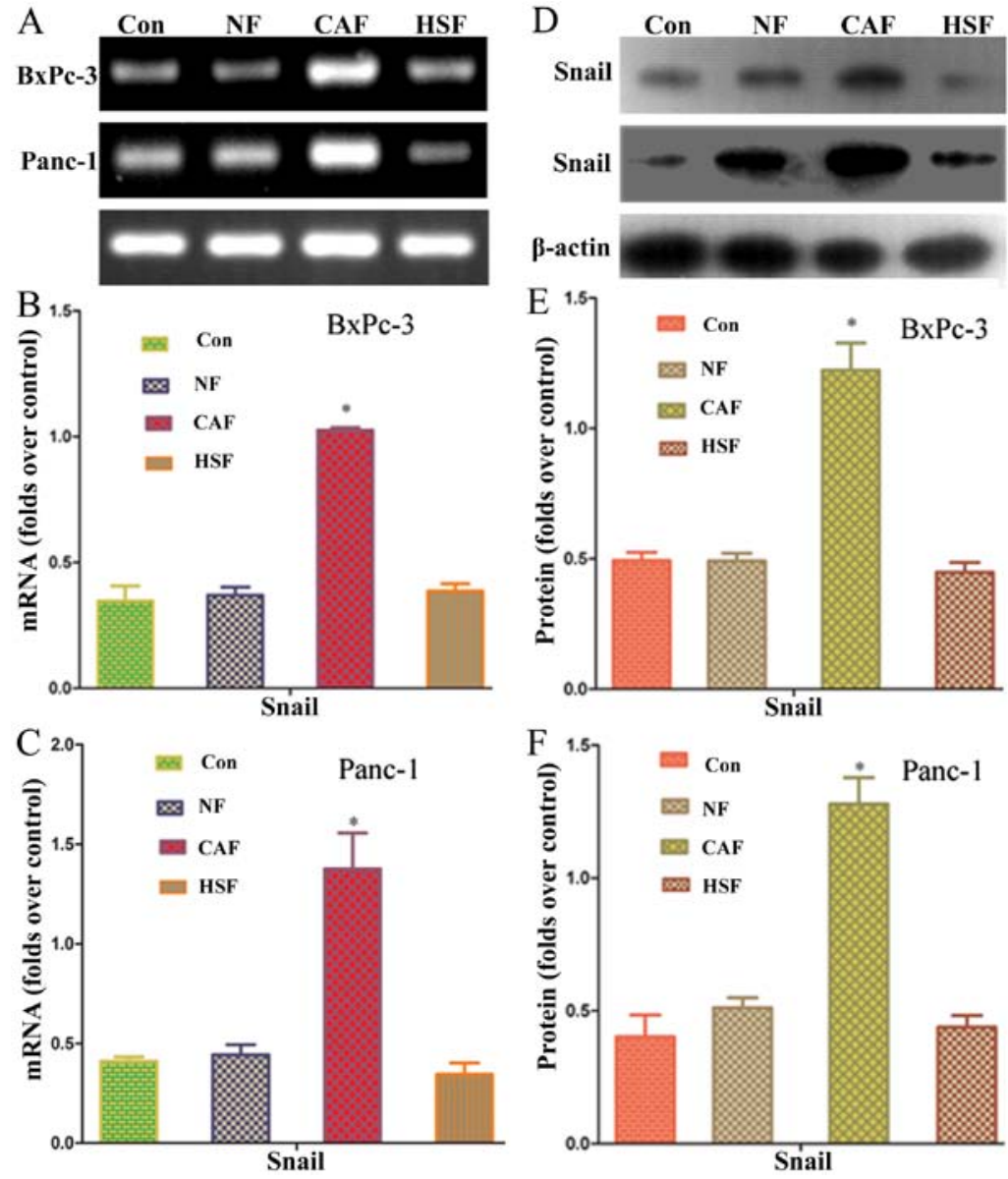

Figure 5. Effects of CAF on Snail mRNA and protein in pancreatic cancer. (A) PCR results showed that the CAF co-culture group had a significantly higher level of Snail mRNA than the NF or HSF co-culture group or single culture group. (B) Results of statistical analysis of Snail mRNA in BxPc-3. (C) Results of statistical analysis of Snail mRNA in Panc-1. (D) Western blotting results showed that the level of Snail protein expression was significantly upregulated as compared with the NF or HSF co-culture group and single culture group. (E) Results of statistical analysis of Snail protein in BxPc-3. (F) Results of statistical analysis of Snail protein in Panc-1. 


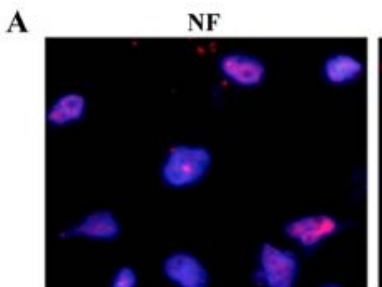

B

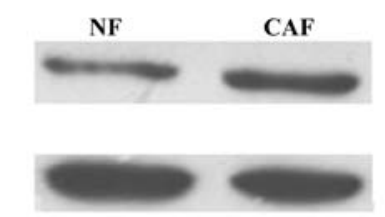

$\mathbf{E}$
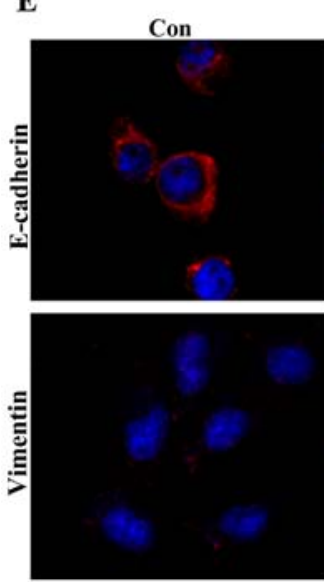
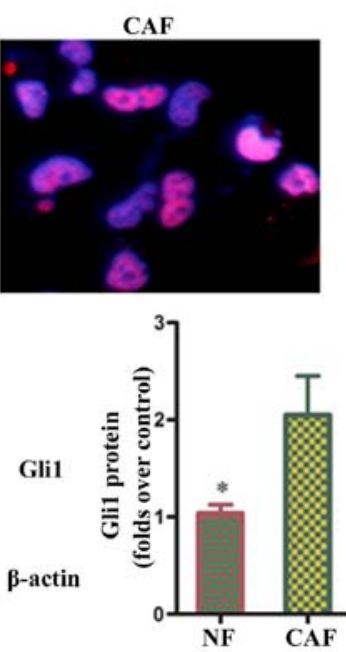
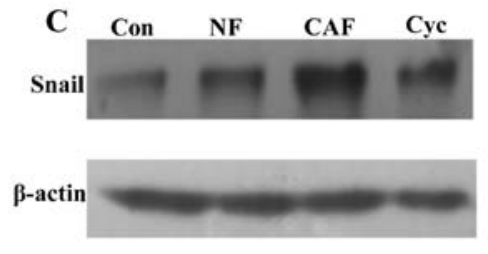

D
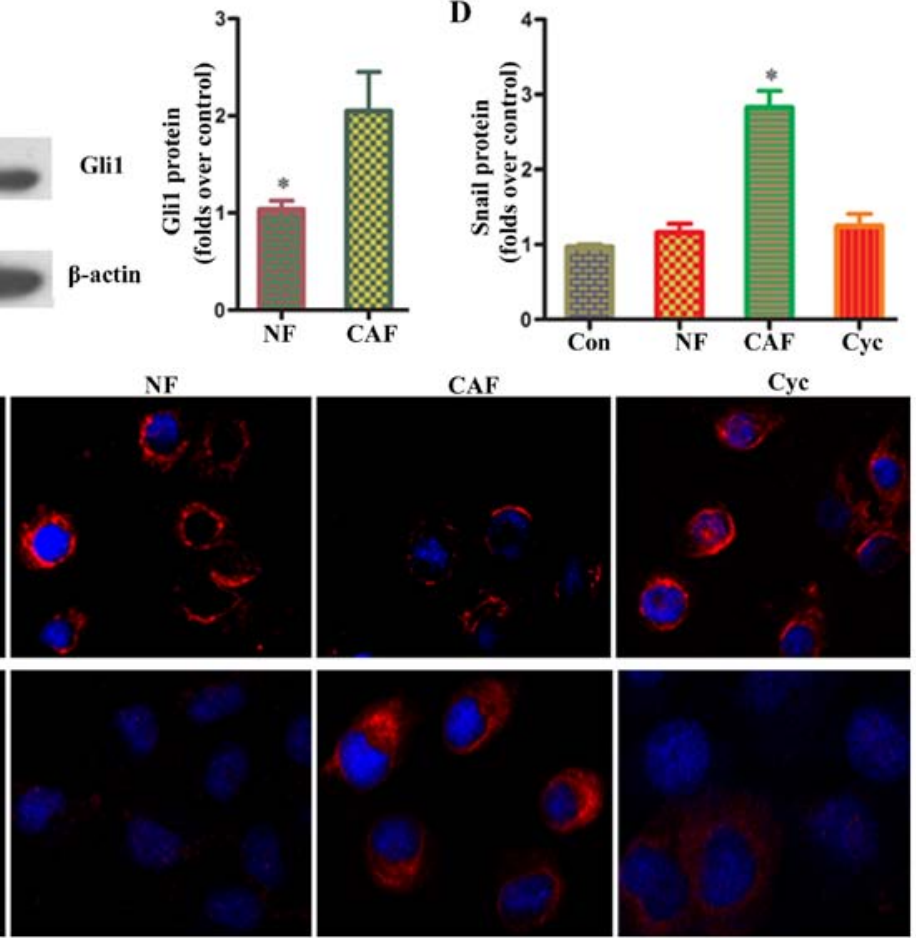

Figure 6. CAF regulates Snail mRNA and protein via the HH pathway in pancreatic cancer cells. (A) Results of Gli1 immunofluorescence showed that CAF had a significantly higher Gli1 fluorescent intensity than the NF group. (B) Western blot analysis showed that Gli1 protein levels were significantly upregulated as compared with the NF group $(\mathrm{P}<0.05)$. CAF was pretreated with an $\mathrm{HH}$-specific blocker (cyclopamine) for $24 \mathrm{~h}$; the supernatant was then obtained and cultured with pancreatic cancer cells. (C and D) Snail protein expression was determined by western blotting. The results showed that Snail protein expression in the cyclopamine group was significantly decreased as compared with the CAF group. (E) The corresponding expression of E-cadherin and vimentin showed significant differences as compared with the CAF group.

Effects of CAF on Snail $m R N A$ and protein in pancreatic cancer. Snail has been reported to regulate EMT marker, E-cadherin, which is a transcription factor upstream of vimentin expression (11). In order to clarify whether pancreatic cancer cells co-cultured with CAF affected Snail expression, RT-PCR and western blotting were used to determine Snail mRNA and protein expressions, respectively. PCR results showed that the CAF co-culture group had a significantly higher level of Snail mRNA than the NF/HSF co-culture group or single culture group $(\mathrm{P}<0.05)$ (Fig. 5). Western blotting results showed that the level of Snail protein expression was significantly upregulated as compared with the NF/HSF co-culture group and single culture group $(\mathrm{P}<0.05)$ (Fig. 5). These results further confirm that Snail expression in pancreatic cancer cells was enhanced after co-culture with CAF.

$C A F$ regulates Snail $m R N A$ and protein via the $H H$ pathway in pancreatic cancer cells. Gli1 have been reported to be terminal transcription regulators of the $\mathrm{HH}$ pathway, and play a very important role in the activation of CAF (12). Activated $\mathrm{CAF}$ can promote tumor progression via paracrine action. In order to clarify whether the upregulation of Snail in pancreatic cancer cells after co-culture is related to the HH pathway in CAF, changes of Gli1 immunofluorescence in CAF were determined using confocal laser scanning microscopy. Results showed that CAF had a significantly higher Glil fluorescent intensity than the NF group (Fig. 6). Western blot analysis showed that Gli1 protein levels were significantly upregulated as compared with the NF group $(\mathrm{P}<0.05)$ (Fig. 6). These results further confirm that the $\mathrm{HH}$ pathway was activated in CAF. To verify whether the HH pathway in CAF was related to EMT in pancreatic cancer cells, CAF was pretreated with an $\mathrm{HH}$-specific blocker (cyclopamine) for $24 \mathrm{~h}$; the supernatant was then obtained and cultured with pancreatic cancer cells. Then, Snail protein expression was determined by western blotting. The results showed that Snail protein expression in the cyclopamine group was significantly decreased as compared with the CAF group, and the corresponding expression of E-cadherin and vimentin showed significant differences as compared with the CAF group. The above results confirm that activating the $\mathrm{HH}$ signaling pathway in CAF may trigger Snail transcription factors in pancreatic cancer cells via paracrine action, thereby enhancing EMT in pancreatic cancer cells. 


\section{Discussion}

In tumor metastasis, EMT promotes cellular migration and invasion to break through the basement membrane (4). It also facilitates the entry of tumor cells into blood circulation, thereby forming CTCs, which promote angiogenesis and intravasation $(13,14)$. Three cell phenotypes exist during the process of EMT, specifically epithelial phenotype (E), mesenchymal phenotype $(\mathrm{M})$ and partial EMT state $(\mathrm{P})$ having both epithelial and mesenchymal features (5). E-cadherin is considered a guardian of epithelial state in various cell types, while vimentin is a canonical molecular marker of EMT events (15). The EMT core transcription factors, Snaill and Snail2, are able to inhibit the transcription of E-cadherin by directly binding to E-boxes on the E-cadherin promoter $(2,15)$. Our study found that activation of Snail transcription factors enhanced EMT, upregulated vimentin expression, and downregulated E-cadherin expression in pancreatic cancer cells, while also enhancing the invasive capacity of pancreatic cancer cells. Our results were consistent with previous studies $(11,16)$.

The occurrence of partial EMT is often not initiated by core tumor cell transcription factors, but instead by the tumor microenvironment (15). In pancreatic carcinomas, CAFs can compose up to $80 \%$ of the tumor mass (8). CAFs can secrete cytokines and growth factors (such as TGF- $\beta$, HGF, FGF, NGF IGF) to enhance epithelial cells migration (17). Various EMT-mediating signaling pathways are cell- or tissue-specific, and interference of EMT may require synergism of multiple signaling pathways, including TGF- $\beta, \mathrm{HH}$, Wnt, and Notch pathways (15). In particular, the TGF- $\beta$ pathway is a major pathway that mediates EMT (18). Previous literature has indicated that TGF- $\beta$ and BMPs induce core EMT transcription factors, Snail1/2, Zeb1/2, and Twist1 $(19,20)$. Fibroblasts in the tumor microenvironment are an important source of TGF- $\beta$ for tumor cells (21). The HH signaling pathway is the key pathway for the development of various tumors by suppressing E-cadherin expression through interactions with Wnt, EGF/ FGF, and TGF- $\beta$ signaling pathways, thereby inducing the onset of EMT and further promoting metastasis (22). The transcription factor of the $\mathrm{HH}$ signaling pathway is Gli1, which has a zinc-finger motif. In the absence of $\mathrm{HH}$ signaling, Gli1 is hydrolyzed into $75-\mathrm{kDa}$ fragments that enter the nucleus and inhibit HH-responsive genes (23). Degradation of Gli1 is inhibited when HH binds to PTCH (23). Full-length Gli1 enters the nucleus and induces the expression of its target genes (23). The HH signaling pathway is closely associated with partial EMT (24). For instance, overexpression of Gli1 by transient transfection of rabbit kidney epithelial cells (RK3E) with low Gli1 expression showed that Snail mRNA expression was upregulated, suggesting that $\mathrm{HH}$ signaling can induce Snail upregulation (25). The $\mathrm{HH}$ signaling pathway can directly upregulate the Notch ligand, JAG2 (26). The binding of JAG2 to Notch activates the Notch signaling pathway that regulates Snail expression (27). The HH signaling pathway may also induce TGF- $\beta$ signaling that further activates MAP3K7 (28). The IKK-NF- $\kappa \mathrm{B}$ signaling pathway upregulates the expression of Snail2 (29). Ma et al reported that activated $\mathrm{HH}$ in CAF can promote the invasion and metastasis of tumor cells via paracrine action (30). Our results showed that $\mathrm{HH}$ in $\mathrm{CAF}$ can regulate Snail expression in tumor cells through paracrine action and control partial EMT in cancer cells, which were consistent with results of previous studies. Most importantly, it was found that $\mathrm{HH}$ signaling in interstitial cells activates partial EMT in adjacent cancer cells, and this mechanism may provide new perspectives in tumor-microenvironment interaction.

The colonization and metastasis of CTCs require the process of $\mathrm{P}$ to $\mathrm{E}$ transition (2). This process, together with the changes in the microenvironment, is closely associated with the changes in the expression of relevant genes at the molecular level. The liver is a common target for the metastasis of pancreatic cancer (31). Our study showed that after an indirect co-culture with HSF, BxPc-3, and Panc-1, cells had significantly higher E-cadherin expression and reduced vimentin expression as compared with the CAF co-culture group, with corresponding reduction in migratory and invasive capacities. PCR and western blotting results showed that both Snail mRNA and protein expression levels in cancer cells were decreased. This suggested that the removal of paracrine action in CAF may be involved in the $\mathrm{P}$ to $\mathrm{E}$ transition of tumor cells, thus enhancing the colonization capacity of tumor cells.

In this study, we demonstrated that activated CAF in the tumor microenvironment may activate the Snail transcription factor in cancer cells through the paracrine action of the $\mathrm{HH}$ signaling pathway. This, therefore, leads to the enhancement of partial EMT in pancreatic cancer cells, upregulation of vimentin expression, downregulation of E-cadherin expression, and enhancement of the invasive capacity of pancreatic cancer cells. We hypothesize that, after CTCs have entered a new homing environment, the paracrine action of normal non-activated fibroblasts downregulate the Gli1-Snail transcriptional regulation axis, leading to P-to-E transition and the formation of new metastatic foci. The metastatic lesion activates CAF, generate partial EMT, which leads to a new cycle, thereby promoting the development of tumors $(31,32)$. These results indicate that the activation of interstitial fibroblasts may be the key to the spatiotemporal regulation of EMT. The reverse process of EMT could become a new preventive approach for the recurrence of tumor cells. Improving our understanding of the molecular regulation of the dynamic EMT process in tumor metastasis may provide us with a more effective way to eradicate tumor metastasis.

\section{Acknowledgements}

This study was supported by the National Natural Science Foundation of China, NSFC (no. 81402583); Natural Science Foundation of Shaanxi Province (no. 2014JQ4165); Xi'an Jiaotong University Education Foundation, XJTUEF (no xjj2014077); and the Hospital Fund of the Second Affiliated Hospital of the Health Science Center, Xi'an Jiaotong University [no. RC (XM)201402)].

\section{References}

1. Winter JM, Cameron JL, Campbell KA, Arnold MA, Chang DC, Coleman J, Hodgin MB, Sauter PK, Hruban RH, Riall TS, et al: 1423 pancreaticoduodenectomies for pancreatic cancer: A single-institution experience. J Gastrointest Surg 10: 1199-1210; discussion 1210-1191, 2006 
2. Tsai JH and Yang J: Epithelial-mesenchymal plasticity in carcinoma metastasis. Genes Dev 27: 2192-2206, 2013.

3. Thiery JP: Epithelial-mesenchymal transitions in tumour progression. Nat Rev Cancer 2: 442-454, 2002.

4. Lim J and Thiery JP: Epithelial-mesenchymal transitions: Insights from development. Development 139: 3471-3486, 2012 .

5. Jordan NV, Johnson GL and Abell AN: Tracking the intermediate stages of epithelial-mesenchymal transition in epithelial stem cells and cancer. Cell Cycle 10: 2865-2873, 2011.

6. de Herreros AG, Peiró S, Nassour M and Savagner P: Snail family regulation and epithelial mesenchymal transitions in breast cancer progression. J Mammary Gland Biol Neoplasia 15: 135-147, 2010.

7. Chui MH: Insights into cancer metastasis from a clinicopathologic perspective: Epithelial-mesenchymal transition is not a necessary step. Int J Cancer 132: 1487-1495, 2013.

8. Olive KP, Jacobetz MA, Davidson CJ, Gopinathan A McIntyre D, Honess D, Madhu B, Goldgraben MA, Caldwell ME, Allard D, et al: Inhibition of Hedgehog signaling enhances delivery of chemotherapy in a mouse model of pancreatic cancer. Science 324: 1457-1461, 2009.

9. Walter K, Omura N, Hong SM, Griffith M, Vincent A, Borges M and Goggins M: Overexpression of smoothened activates the sonic hedgehog signaling pathway in pancreatic cancer-associated fibroblasts. Clin Cancer Res 16: 1781-1789, 2010.

10. Walter K, Omura N, Hong SM, Griffith M and Goggins M: Pancreatic cancer associated fibroblasts display normal allelotypes. Cancer Biol Ther 7: 882-888, 2008.

11. Cano A, Pérez-Moreno MA, Rodrigo I, Locascio A, Blanco MJ, del Barrio MG, Portillo F and Nieto MA: The transcription factor snail controls epithelial-mesenchymal transitions by repressing E-cadherin expression. Nat Cell Biol 2: 76-83,2000.

12. Rimkus TK, Carpenter RL, Qasem S, Chan M and Lo HW: Targeting the sonic hedgehog signaling pathway: Review of smoothened and GLI inhibitors. Cancers (Basel) 8: 8, 2016.

13. Kallergi G, Papadaki MA,Politaki E, Mavroudis D, Georgoulias V and Agelaki S: Epithelial to mesenchymal transition marker expressed in circulating tumour cells of early and metastatic breast cancer patients. Breast Cancer Res 13: R59, 2011

14. Ota I, Li XY, Hu Y and Weiss SJ: Induction of a MT1-MMP and MT2-MMP-dependent basement membrane transmigration program in cancer cells by Snaill. Proc Natl Acad Sci USA 106 20318-20323, 2009.

15. De Craene B and Berx G: Regulatory networks defining EMT during cancer initiation and progression. Nat Rev Cancer 13: 97-110, 2013

16. Batlle E, Sancho E, Francí C, Domínguez D, Monfar M, Baulida J and García De Herreros A: The transcription factor snail is a repressor of E-cadherin gene expression in epithelial tumour cells. Nat Cell Biol 2: 84-89, 2000.
17. Gascard P and Tlsty TD: Carcinoma-associated fibroblasts: Orchestrating the composition of malignancy. Genes Dev 30: $1002-1019,2016$

18. Katsuno Y, Lamouille S and Derynck R: TGF- $\beta$ signaling and epithelial-mesenchymal transition in cancer progression. Curr Opin Oncol 25: 76-84, 2013.

19. Eckert MA, Lwin TM, Chang AT, Kim J, Danis E, OhnoMachado L and Yang J: Twistl-induced invadopodia formation promotes tumor metastasis. Cancer Cell 19: 372-386, 2011.

20. Thiery JP, Acloque H, Huang RY and Nieto MA: Epithelialmesenchymal transitions in development and disease. Cell 139: 871-890, 2009.

21. Liu FL, Mo EP, Yang L, Du J, Wang HS, Zhang H, Kurihara H, $\mathrm{Xu} \mathrm{J}$ and Cai SH: Autophagy is involved in TGF- $\beta 1$-induced protective mechanisms and formation of cancer-associated fibroblasts phenotype in tumor microenvironment. Oncotarget 7 : 4122-4141, 2016.

22. Zhang J, Tian XJ and Xing J: Signal transduction pathways of EMT induced by TGF- $\beta$, SHH, and WNT and their crosstalks. J Clin Med 5: 5, 2016.

23. Carpenter RL and Lo HW: Hedgehog pathway and GLI1 isoforms in human cancer. Discov Med 13: 105-113, 2012.

24. Palle K, Mani C, Tripathi K and Athar M: Aberrant GLI1 Activation in DNA damage response, carcinogenesis and chemoresistance. Cancers (Basel) 7: 2330-2351, 2015.

25. Li X, Deng W, Nail CD, Bailey SK, Kraus MH, Ruppert JM and Lobo-Ruppert SM: Snail induction is an early response to Gli1 that determines the efficiency of epithelial transformation. Oncogene 25: 609-621, 2006.

26. Visbal AP, LaMarca HL, Villanueva H, Toneff MJ, Li Y, Rosen JM and Lewis MT: Altered differentiation and paracrine stimulation of mammary epithelial cell proliferation by conditionally activated Smoothened. Dev Biol 352: 116-127, 2011.

27. Katoh Y and Katoh M: Hedgehog target genes: Mechanisms of carcinogenesis induced by aberrant hedgehog signaling activation. Curr Mol Med 9: 873-886, 2009.

28. Perrot CY, Javelaud D and Mauviel A: Overlapping activities of TGF- $\beta$ and Hedgehog signaling in cancer: Therapeutic targets for cancer treatment. Pharmacol Ther 137: 183-199, 2013.

29. Ren X, Wang F, Ji B and Gao C: TLR7 agonist induced repression of hepatocellular carcinoma via the TLR7-IKK-NF- $\kappa B-I L 6$ signaling pathway. Oncol Lett 11: 2965-2970, 2016.

30. Ma J, Cheng J, Gong Y, Tian L and Huang Q: Downregulation of Wnt signaling by sonic hedgehog activation promotes repopulation of human tumor cell lines. Dis Model Mech 8: 385-391, 2015.

31. Shi H, Li J and Fu D: Process of hepatic metastasis from pancreatic cancer: Biology with clinical significance. J Cancer Res Clin Oncol 142: 1137-1161, 2016.

32. Pantel K and Brakenhoff RH: Dissecting the metastatic cascade. Nat Rev Cancer 4: 448-456, 2004. 